\title{
Monitoring of impact damage in Carbon Fibre Reinforced Polymers
}

\author{
by C. Meola, G. M. Carlomagno and F. Ricci
}

Dept. of Aerospace Engineering, University of Naples, Via Claudio 21-80125 Napoli, Italy, carmeola@unina.it

\begin{abstract}
Infrared thermography is utilized to gain new information on onset and propagation of impact damage in Carbon Fibre Reinforced Polymers. Impact tests are carried out with a modified Charpy pendulum while the SC6000 camera views the specimen surface opposite to impact and records sequences of thermal images at $96 \mathrm{~Hz}$. The same infrared camera, equipped with the lock-in option, is also used for non-destructive evaluation of the specimens before and after impact; results are shown as phase images. The elastic waves propagating from the impact are also recorded with piezo patches. A good agreement is found regarding the damage onset.
\end{abstract}

\section{Introduction}

Carbon fibres are widely used as reinforcing elements in the production of composite materials. Carbon Fibre Reinforced Polymers CFRP are finding increasing use as primary structural components in many modern applications due to their high strength-to-weight ratio, formability and other properties that make them preferable to metals or other conventional engineering materials. However, as in general all types of composites, they exhibit different problems when compared to metallic materials, in terms of type and occurrence of flaws from their production to their in-service life.

The duration of a metallic component is dependent on the possible formation of a crack and its growth. Metal fracture mechanics is often adequate to predict the size of critical flaws and, as a consequence, to establish rejection/acceptance criteria on the basis of the designer requirements. On the contrary, CFRPs are strongly inhomogeneous and their behaviour is complex and difficult to be modelled. In fact, a lot of models have been developed to describe the variety of possible defects such as interlaminar deboding, matrix degradation, fibre rupture and total or partial separation of the matrix and the fibres [1]. At the moment, all these models seem to be unable to completely describe the complexity of the failure starting point and its propagation in composites so that, the availability of experimental data is of great importance. In this context, infrared thermography represents a useful investigation tool. It was recently used by Meola and Carlomagno [2] who supplied information on onset and propagation of impact damage in Glass Fibres Reinforced Polymers (GFRP) through the analysis of thermoplastics effects developing under impact. Most recently, Meola et al. [3] extended the application of infrared thermography to monitor different types of composites under impact tests.

The aim of this work is to go on in the investigation of impact damage in CFRP with infrared thermography and to search for new information on the behaviour of such materials to an impact event. To this end, the surface temperature measurements, for some tests, are also compared with the elastic waves propagating from the impact zone, which are recorded with piezo-patches.

\section{Materials and test setup}

Two types of specimens are considered:

- $\quad$ Carbon fibre reinforced polymer (CFRP) obtained from unidirectional carbon fibres embedded in epoxy resin were fabricated by the hand lay-up technology and cured in autoclave. Specimens are $130 \times 100 \mathrm{~mm}^{2}$ and 2.4 mm thick and are named $C_{u}$.

- $\quad$ Cross ply carbon fibre reinforced polymer (CFRP) of stacking sequence [0/90 $]_{4}$ which is $555 \mathrm{~mm}$ long, $250 \mathrm{~mm}$ wide and $2.5 \mathrm{~mm}$ thick; this specimen is named $C_{c p}$.

Specimens are first non-destructively inspected with lock-in thermography to search for manufacturing defects. Then, each specimen is impacted at a fixed energy while the infrared camera views its rear side. Finally, each impacted specimen is inspected again with lock-in thermography.

Impact tests are carried out with a modified Charpy pendulum including an impactor of hemispherical nose 24 $\mathrm{mm}$ in diameter. In figure 1 it is shown a) the sketch of the panel with the impact location and piezo patches positions b) the position of the infrared camera to see the specimen's rear side and c) one specimen inside the lodge. Specimens are held from the shorter sides, while are free to move along the two longer sides. The impact energy $E_{i}$ is varied in the range $0.5-2.8$ Joule by varying the starting height of the Charpy arm. The impact location on the panel has been changed for each impact event in order to evaluate the impact effect on a damage free zone.

The used infrared camera is the SC6000 (Flir systems), which is equipped with a QWIP detector working in the 8-9 $\mu \mathrm{m}$ infrared band, has a $50 \mathrm{~mm}$ focal length lens, a spatial resolution $640 \times 512$ pixels full frame and a windowing 
option linked to frequency frame rate and temperature range. The same camera, equipped with the Lock-in option and the IrNDT software, is used for non-destructive tests.

In order to monitor the elastic waves propagating from the impact point [4], some piezo patches are bonded on the impact surface of the specimen $C_{o p}$ as Figure.1 a) and c) show.

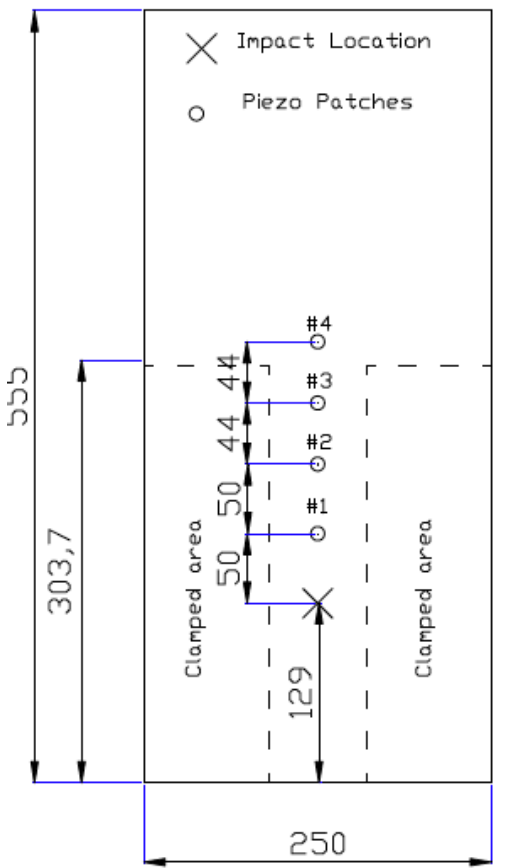

a) Sketch of the panel with impact location and piezo patches positions

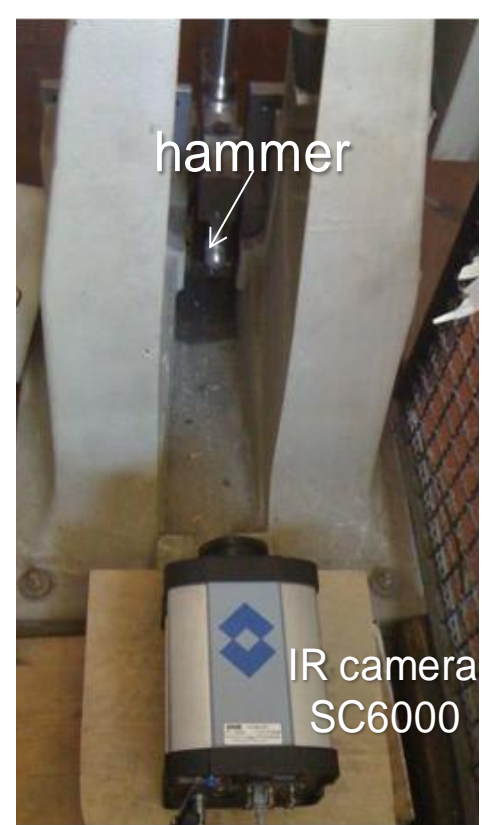

b) position of the infrared camera

Fig. 1. Impact test setup

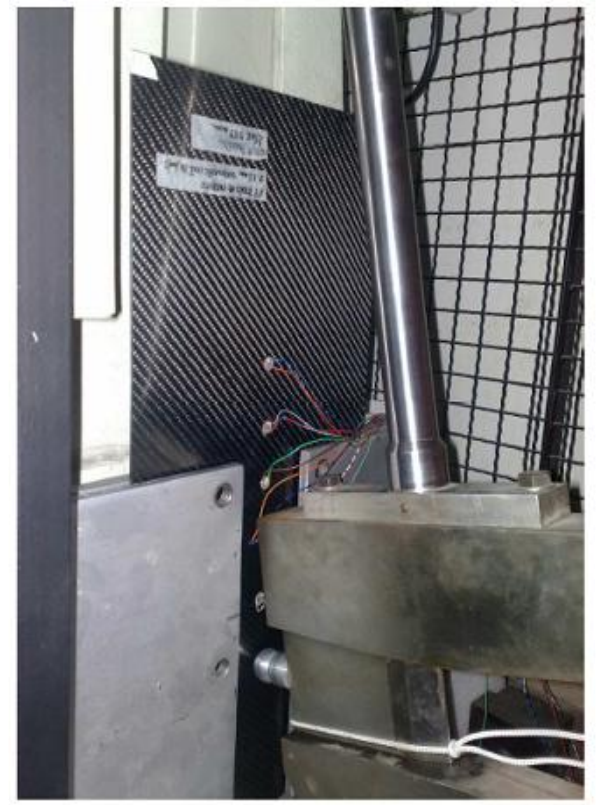

c) view of the specimen setup

\section{On-line surface temperature mapping}

Sequences of thermal images are acquired at $96 \mathrm{~Hz}$, with the SC6000 infrared camera, during impact tests. More specifically, the acquisition starts few seconds before the impact and lasts for some time after to allow complete visualization of thermal effects evolution with respect to the ambient temperature. In fact, on the surface opposite to the hammer's contact, the material undergoes first cooling down due to thermo-elastic effects and then heating up because of the dissipation of the impact mechanical energy. It has to be considered that temperature variations occur during a very short time (small fractions of seconds), and thus, their visualization is possible only with a high-frequency imaging device; in particular, thermo-elastic effects were visualized and analysed for the case of GFRP by Meola and Carlomagno in a previous work through acquisition of images at $900 \mathrm{~Hz}$ [5] with the SC3000 infrared camera; in that case, the image included only 16 horizontal lines and so a small specimen portion was visualized. In the present paper, the attention is mainly focused on the heating phase since detection of the heat generation loci is important for the comprehension of initiation and propagation of the damage mechanisms. The visualized thermoplastic phase is, de facto, slower than the thermo-elastic one and then, it is possible to gain information also through acquisition of images at the lower frequency of $96 \mathrm{~Hz}$. This offers the advantage of viewing a surface area wide enough to account for the in-plane extension of the damage.

To better analyse the thermal behaviour, the first image ( $t=0 \mathrm{~s}$ ) of the sequence, i.e. the specimen surface (ambient) temperature before the impact, is subtracted to each subsequent image so as to generate a map of the temperature difference $\Delta T$ given by Eq (1):

$$
\Delta T=T(i, j, t)-T(i, j, 0)
$$

$i$ and $j$ representing lines and columns of the surface temperature map. Therefore, a sequence of $\Delta T$ images is created.

Some examples of $\Delta T$ images are shown in the following figures 2-5 involving both types of specimens and three different values of the impact energy $E_{i}=0.5,2.3$ and $2.8 \mathrm{~J}$. More specifically, figure 2 shows thermal images of both $C_{c p}$ (Fig.2a) and $C_{u}$ (Fig.2b) specimens, which were taken during the contact of the surface with the hammer for $E_{i}=$ $2.8 \mathrm{~J}$. Instead figure 3 compares thermal images of the specimen $C_{c p}$ taken soon at the contact with the hammer (Fig.3a) and $0.01 \mathrm{~s}$ later (Fig.3b) for $E_{i}=0.5 \mathrm{~J}$. Thermal images of the specimen $C_{c p}$, taken $0.01 \mathrm{~s}$ after impact, are shown in 
figure 4, for $E_{i}=2.3 \mathrm{~J}$ (Fig.4a) and for $E_{i}=2.8 \mathrm{~J}$ (Fig.4b). Figure 5 shows thermal images of the specimen $C_{u}$, taken 0.01 (Fig.5a) and $0.8 \mathrm{~s}$ (Fig.5b) after impact for $E_{i}=2.8 \mathrm{~J}$, as normal view (Fig.5a) and magnification of the hot zone on the top right side. Finally, figure 6 shows again thermal images of the specimen $C_{c p}$, but taken some time later after impact.

First of all, it has to be noted the presence of cooling zones (negative $\Delta T$ values) in figures 2 and $3 a$; this indicates presence of thermo-elastic effects, which evolve in a very fast way and were analysed through acquisition of images at $900 \mathrm{~Hz}$ [5]; but some effects are herein visualized also with sampling rate of $96 \mathrm{~Hz}$. As a general comment, the cooling-down zone assumes a circular shape (see Fig. 2 and $3 a$ ), which is practically independent of the laminate stacking sequence and of the impact energy. Actuality, the temperature decrease is inversely proportional to the impact energy [5], but its shape geometry depends essentially on the size of the hammer nose; on the other hand, the thermoelastic effect is caused by the material expansion, which, in turn depends on the surface bending that is proportional to the pushing object size. Conversely, from figures 3b, 4 and 5 , and by considering also previously published data for glass reinforcing fibres $[2,5]$, it is possible to demonstrate the strong influence of the type of reinforcing material as well the stacking sequence on the way the heat, produced by the impact energy, is dissipated.

In fact, a slighter zone composed of only a few pixels is interested by heating up for the $C_{c p}$ specimen when it is impacted at $E_{i}=0.5 \mathrm{~J}$; but, as the impact energy overpasses $2 \mathrm{~J}$, the hot zone evolves in a different manner depending on the impact energy and principally on the material type. From figure $4 \mathrm{a}$, which refers to the $C_{c p}$ specimen impacted at $E_{i}$ $=2.3 \mathrm{~J}$, it is possible to see a lighter structure, emerging from the darker background and developing mainly in vertical direction as a narrow stripe with branches on the right side. In particular, the vertical length is $21 \mathrm{~mm}$, while the horizontal branch is $4.8 \mathrm{~mm}$ wide and the oblique branch $3.6 \mathrm{~mm}$ long; the hottest central part, considering both vertical and oblique orientations is $8.5 \mathrm{~mm}$. For $E_{i}=2.8 \mathrm{~J}$ (Fig.4b), the hot structure takes the appearance of a perfect cross, which extends for about 15 and $25 \mathrm{~mm}$ respectively along horizontal and vertical directions. Conversely, the specimen $C_{u}$ displays, at the same energy $E_{i}=2.8 \mathrm{~J}$, an about $8 \mathrm{~mm}$ long hot structure (Fig.5a), in which, looking at the magnification on the right upside, it is possible to recognize a train of hot short vertical lines. Such lines are characterized by a pitch of 3 pixels (about $0.8 \mathrm{~mm}$ ). To a watchful eye, also the hot structures, on the $C_{c p}$ specimen, appear composed of alternating lines at a pitch of 1 pixel (about $0.27 \mathrm{~mm}$ ) along both horizontal and vertical directions. To explain this, we have to consider first that:

- we are dealing with low velocity impact on relatively thin plates (2.4 or $2.5 \mathrm{~mm}$ thick), for which the damage should begin on the side opposite to the knock (i.e. the stretched side viewed by the camera);

- $\quad$ most of the absorbed energy is dissipated as heat;

- from previous studies [6-8], there is a general believe that, for low velocity impact, the damage process is initiated by matrix cracks, which form under surface bending.

And then, it is possible to infer that the slighter warming up, visible on the $C_{c p}$ specimen surface for $E_{i}=0.5 \mathrm{~J}$ (Fig. 3b), is caused by the formation of micro-cracks, which, of course, is accompanied by a very small dissipation of energy. For increasing the impact energy, the amount of energy which is converted into heat increases too as due to the development of micro-cracks into delamination. Certainly, the preferred pathway for delamination is along adjacent fibres. Thus, for the $C_{u}$ specimen, entailing unidirectional fibres a $0^{\circ}$ and $90^{\circ}$, it is apparent that delamination occurs along a horizontal line since, on the viewed surface, the first layer includes fibres at $0^{\circ}$. A certain amount of delamination affects also the second deeper layer with fibres at $90^{\circ}$, but for a shorter path; most probably, because of stress attenuation with the depth. However, this hot zone tends to enlarge with time (Fig.5b) and to become much milder in terms of temperature difference $\left(\Delta T_{\max }=1.5^{\circ} \mathrm{C}\right)$ with respect to the ambient because of heat transfer inside the material.

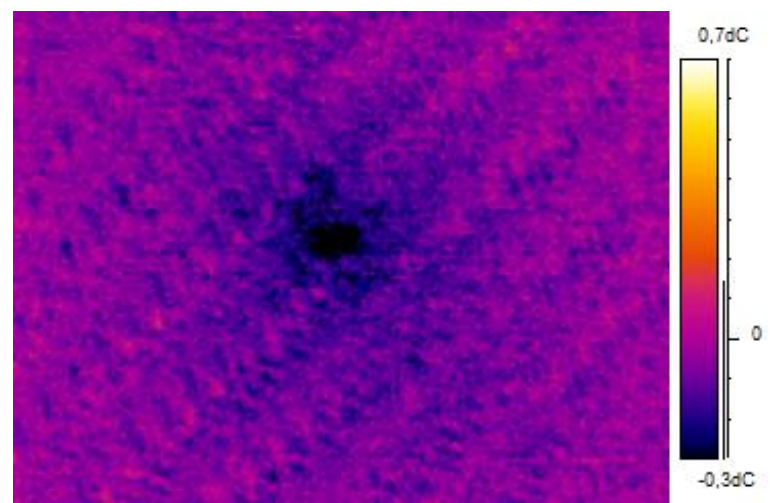

a) $C_{c p}$ specimen

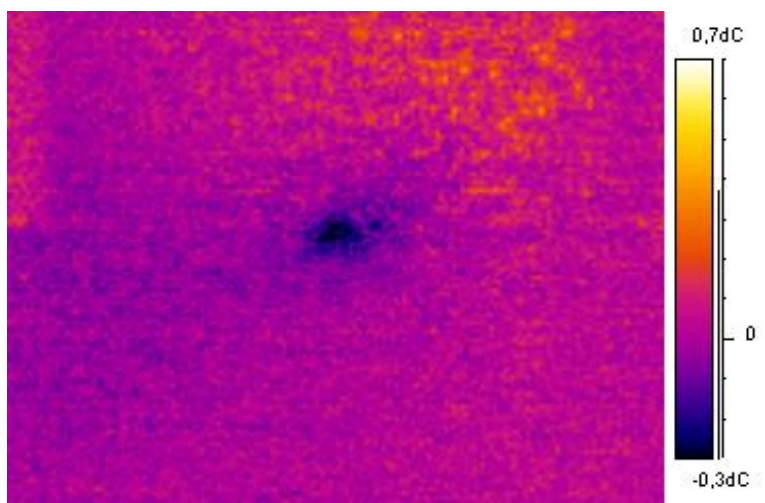

b) $C_{u}$ specimen

Fig. 2. $\Delta T$ images taken at the impact for $E_{i}=2.8 \mathrm{~J}$

Of more difficult interpretation appears, at first sight, the cross-like structure in figure 4b. However, in the case of a cross ply laminate, such as the $C_{c p}$ specimen, there is not a preferred pathway, but delamination occurs, equally allocated along both $0^{\circ}$ and $90^{\circ}$ directions; the only difference is the length that is longer in the vertical direction. The 
latter is to be ascribed to the influence of the grasping force which is perpendicular to the viewed horizontal direction. Again, with time, the hot zone tends towards a circular one of lower temperature difference, because of the aforementioned heat transfer within the material. In particular, figure 6 shows the thermal images of specimen $C_{c p}$ impacted at $2.3 \mathrm{~J}$ (Fig.6a) and at $2.8 \mathrm{~J}$ (Fig.6b) taken when $\Delta T_{\max }=1.5{ }^{\circ} \mathrm{C}$, which happens after 4.6 and $6.8 \mathrm{~s}$ respectively. Of course, the $\Delta T_{\max }$ value is reached at about the centre of the circle.

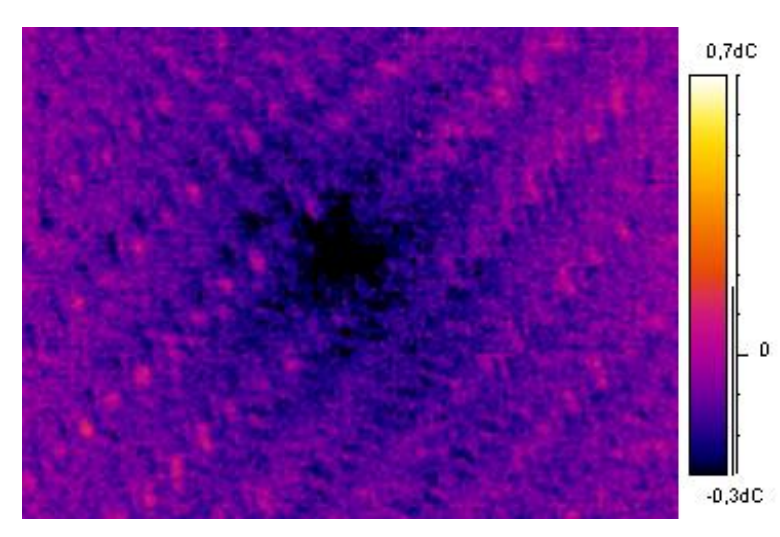

a) at the impact

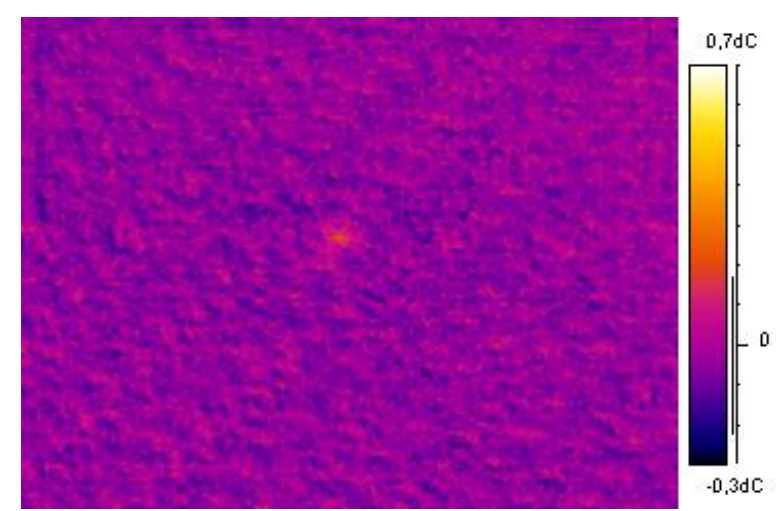

b) 0.01 s later

Fig. 3. $\Delta T$ images of $C_{c p}$ specimen for $E_{i}=0.5 \mathrm{~J}$

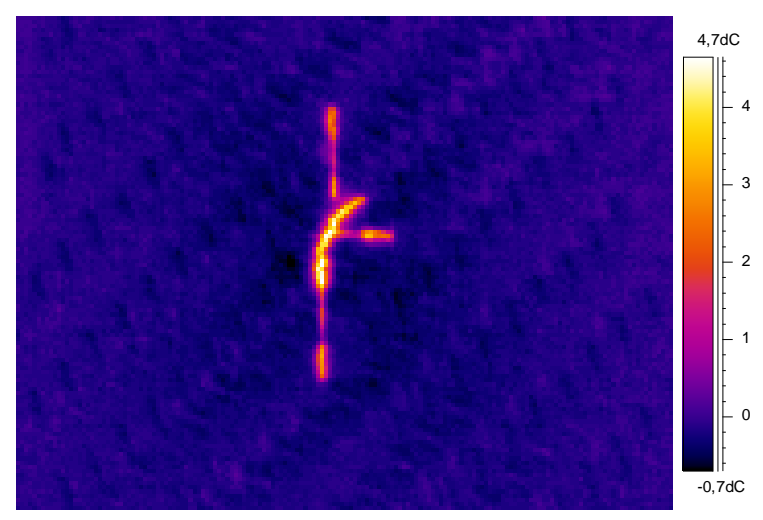

a) $E_{i}=2.3 \mathrm{~J}$

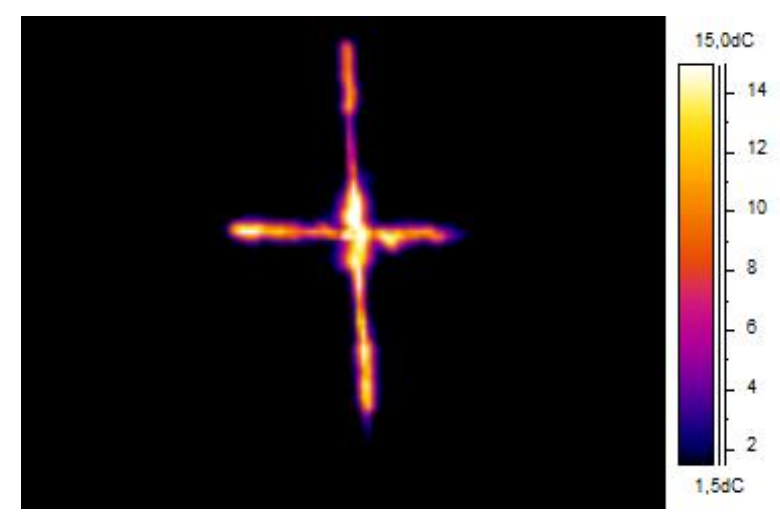

b) $E_{i}=2.8 \mathrm{~J}$

Fig. 4. $\Delta T$ images of the $C_{c p}$ specimen taken $0.01 \mathrm{~s}$ after impact

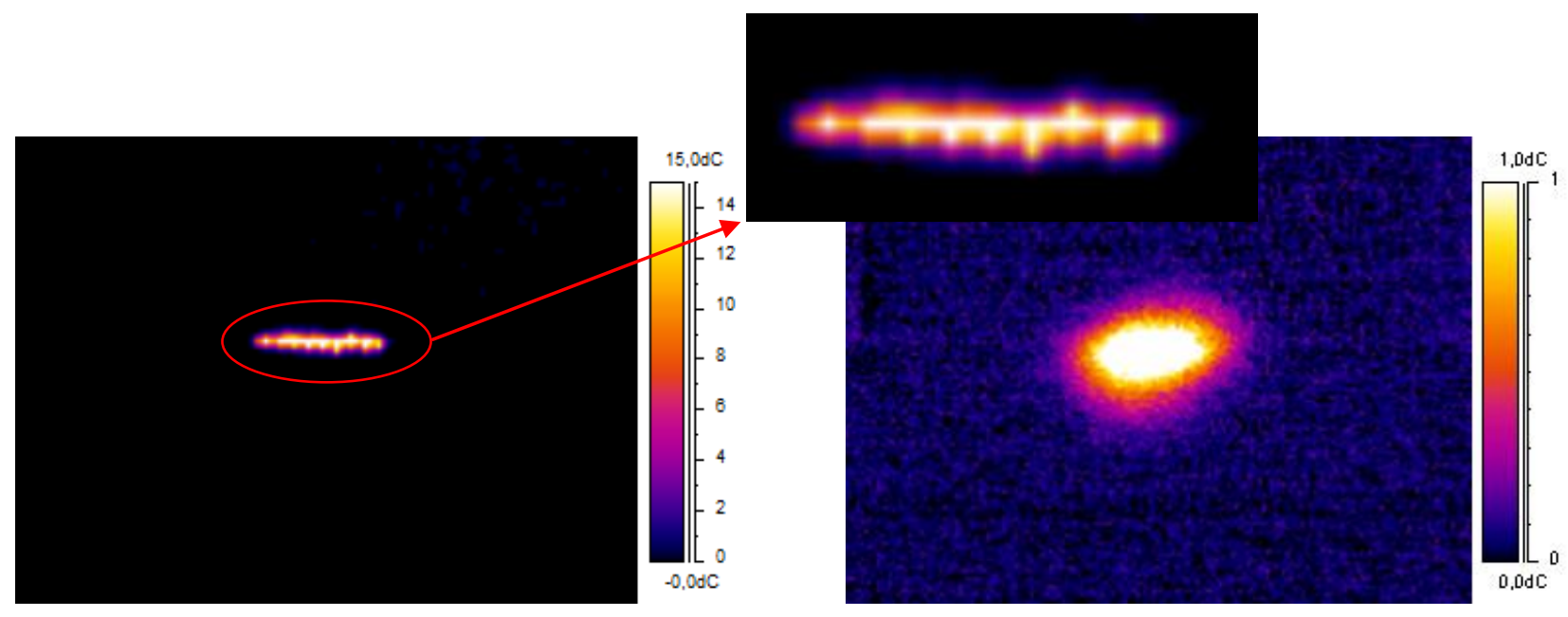

a) $0.01 \mathrm{~s}$ after impact

b) $0.8 \mathrm{~s}$ after impact

Fig. 5. $\Delta T$ images of the $C_{u}$ specimen for $E_{i}=2.8 \mathrm{~J}$ 


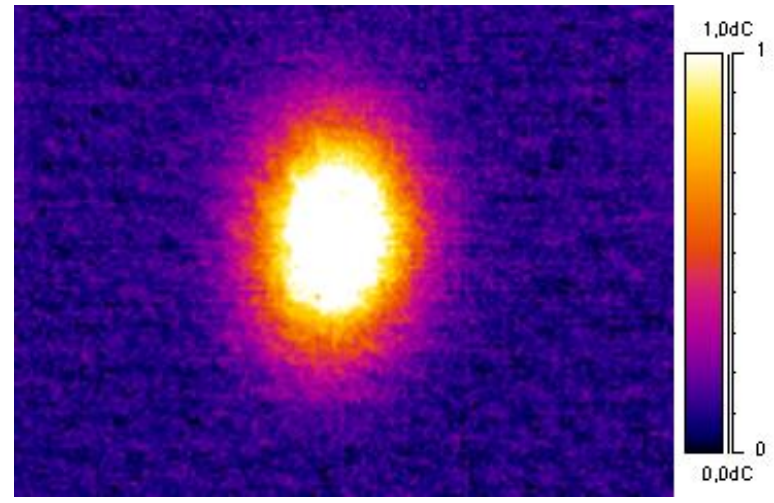

a) $E_{i}=2.3 \mathrm{~J}, t=4.6 \mathrm{~s}$ after impact
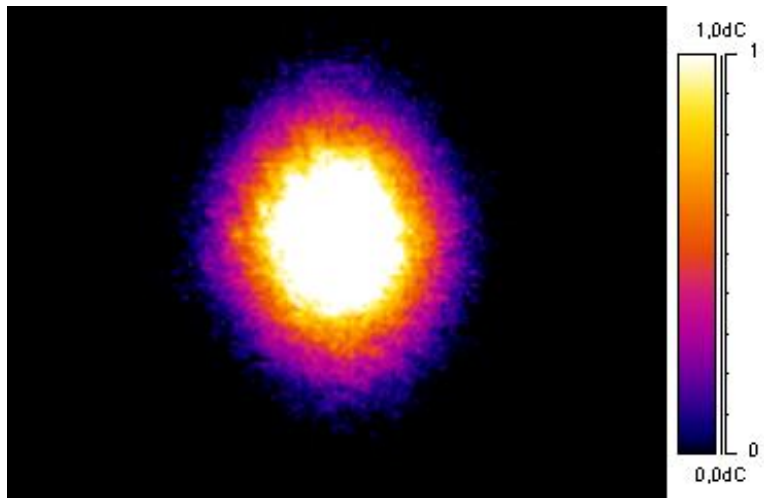

b) $E_{i}=2.8 \mathrm{~J}, t=6.8 \mathrm{~s}$ after impact

Fig. 6. $\Delta T$ images of the $C_{c p}$ specimen taken for $\Delta T_{\max }=1.5^{\circ} \mathrm{C}$

\subsection{Comparison between temperature increase and elastic energy propagation}

The passive impact detection technique using piezo patches bonded to the specimen surface consists in the analysis of the signals collected by an array of piezo sensors capable to detect the elastic waves propagating through the structure from the impact point. The analysis of such signals might give important information on the impact event, and in particular on the impact location as well as on the extension and severity of the impact damage.

The signal from piezo sensors is recorded during impact tests, on the $C_{c p}$ specimen, by varying the impact energy from 0.5 up to $2.8 \mathrm{~J}$ [9]. Three plots for $E_{i}=0.5,1.2$ and $2.8 \mathrm{~J}$ are shown in figure 7 (a-c); while the maximum signal $S A_{\max }$, recorded from the piezo patch \#2, for each impact energy is reported in figure $7 \mathrm{~d}$.

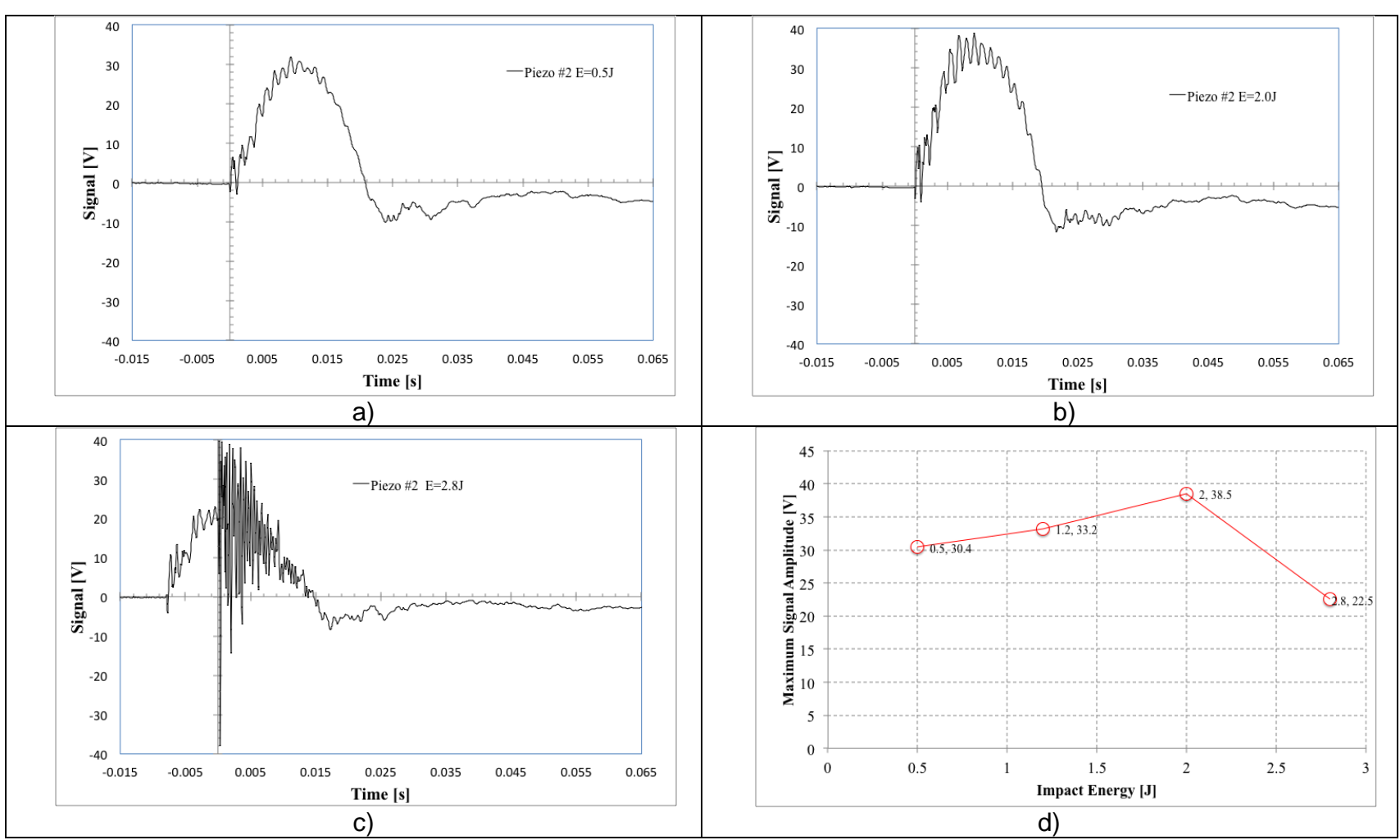

Fig. 7. Piezo sensor signals during impacts at a) $0.5 \mathrm{~J}, b) 2.0=\mathrm{J}$ and c) $2.8 \mathrm{~J}$. Figure d) report the maximum signal recorded from the piezo patch \#2 
It is observed an almost monotonic increase of the signal till $E_{i}=2.0 \mathrm{~J}$; in particular, the signal assumes a quasismoothed shape (Fig.7a). Instead, for $E_{i}=2.8 \mathrm{~J}$, as shown in figure 7c, the recorded signal is very noisy characterized by high frequency oscillations and by a decrease in amplitude with respect to that obtained for $E_{i}=2.0 \mathrm{~J}$ (Fig.7d); this means that some impact damage occurred that generates multiple acoustic emissions.

For a direct comparison between the two different used techniques: piezo sensors and infrared thermography, values of $\Delta T_{\max }$ and $S A_{\max }$ are plotted against the impact energy in figure 8. A good agreement between the two types of data is achieved.

In fact, till $E_{i}=2.0 \mathrm{~J}, \Delta T_{\max }$ is close to zero to mean, as already observed in the previous section, that a very small, almost irrelevant, amount of impact energy has been dissipated into heat. This, in turn, means that no important damage occurred, but only micro-cracks in the matrix. This may be regarded as the initiation phase of the impact damage [6-8], as, on the other hand, was already demonstrated through the use of infrared thermography within impact tests on GFRP specimens [2]. However, the presence of micro-cracks in the matrix does not alter the acoustic characteristics of the material. In fact, till $E_{i}=2.0 \mathrm{~J}$, the amount of energy spent in acoustic emission and recorded by piezo patches increases proportionally to the increase of the impact energy that bears witness for an overall integrity of the material.

For $E_{>}>2.0 \mathrm{~J}$, the amount of energy absorbed as heat increases abruptly to over $20 \mathrm{~K}$ to mean that important damage has occurred most probably fibre breakage and/or important delamination between layers (Fig.4). This is corroborated by the alteration in acoustic emission (Fig.7c). In particular, for $E_{i}>2.8 \mathrm{~J}$, the amplitude of the acoustic signal falls down abruptly from the value of about $40 \mathrm{~V}$, which was attained for $E_{i}>2.0 \mathrm{~J}$, to a value nearby $20 \mathrm{~V}$ (Fig.7d); this means that some damage occurred in the material. Therefore, from the diagram in figure 8 , it is possible to assume $E_{i}=2.0 \mathrm{~J}$ as limiting value between initiation and propagation phases of the impact damage for the analysed type of composite material.

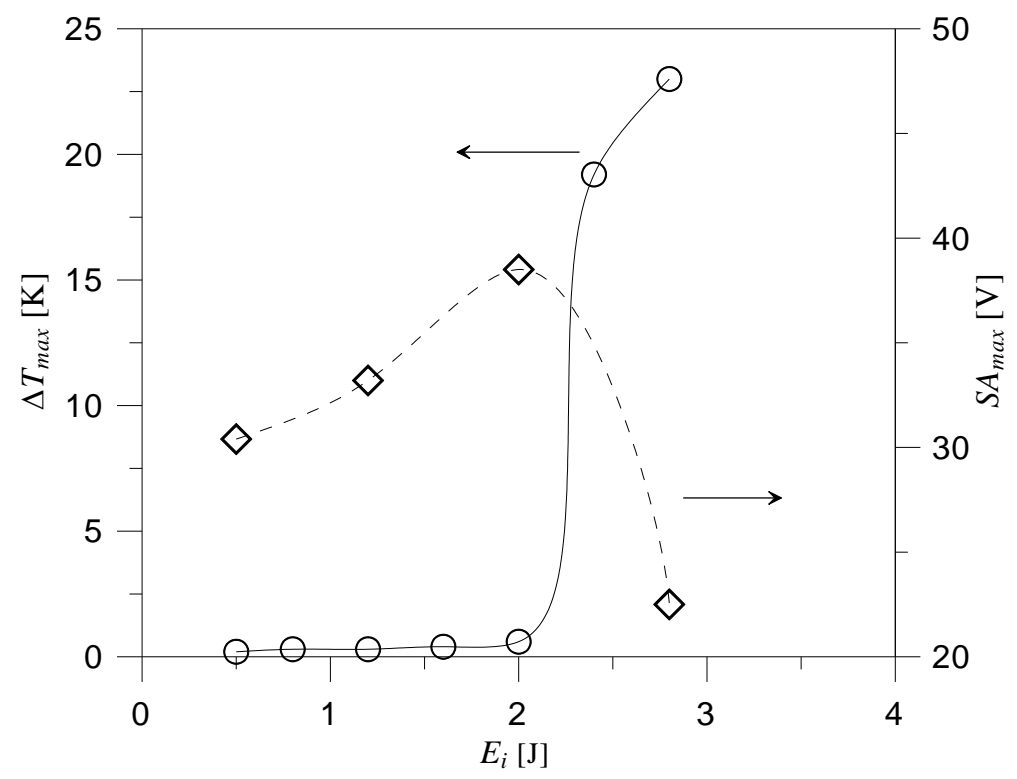

Fig. 8. Plot of $\Delta T_{\max }$ and elastic signal amplitude against $E_{i}$ for the $C_{c p}$ specimen

\section{Non-destructive testing}

Non-destructive tests are performed with lock-in thermography and optical stimulation with one, or two halogen lamps $1 \mathrm{~kW}$ each (depending on the specimen width). As an example, in figure 9 are shown two phase images, taken at the heating frequency of $0.075 \mathrm{~Hz}$, of the $C_{c p}$ specimen, which was impacted in two different zones respectively at $E_{i}=$ 2.3 and $2.8 \mathrm{~J}$.

Firstly, it is worth noting that the white circular zones indicate the presence of one piezo, which is attached on the rear side (see Fig.1b). It is possible to see light structures (encircled in figure), which recall those already visualized during online monitoring (Fig.4a and b). More specifically, the first structure (Fig. 9a) has the appearance of a stripe that is oblique-vertically oriented; its length is about $8 \mathrm{~mm}$. The other one (Fig.9b) appears as a cross of branches respectively $11.4 \mathrm{~mm}$ and $18 \mathrm{~mm}$ long along horizontal and vertical directions.

As a general comment, it seems the damage, detected through non-destructive testing, does not match the hot signature observed during online monitoring. in fact, by comparing figure $7 \mathrm{a}$ to figure $4 \mathrm{a}$ it is possible to see that only the hottest oblique structure part is detected in the phase image. Analogously, the slender upper part of the cross, clearly 
visible in figure $4 \mathrm{~b}$, is lost in figure $9 \mathrm{~b}$. From phase images is not possible to see the entire extension of the cross vertical and horizontal lines. This because, as can be seen from the thermal image (Fig.4b), during the impact, delamination extended through thin lines, which are about 0.3 to $0.6 \mathrm{~mm}$ wide and characterized by slight temperature increase (the $\Delta T$ goes up to $20^{\circ} \mathrm{C}$ in the centre and down to $2{ }^{\circ} \mathrm{C}$ in the slender parts). Most probably, the slender parts indicate slight delamination, which does not cause significant perturbation to the phase angle map and gets lost within the measurement noise.

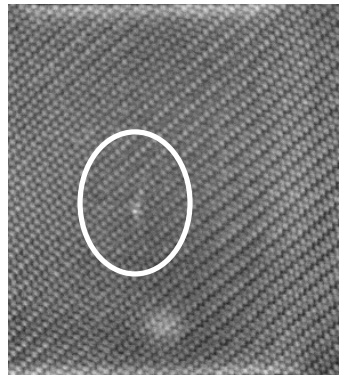

a) $E_{i}=2.3 \mathrm{~J}$

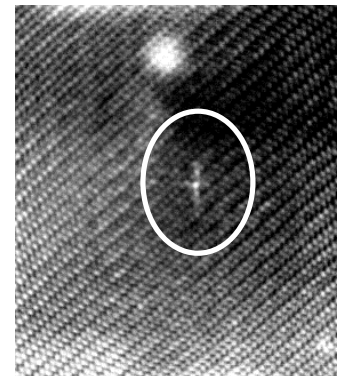

b) $E_{i}=2.8 \mathrm{~J}$

Fig. 9. Phase images taken at $f=0.075 \mathrm{~Hz}$ of $C_{c p}$ specimen impacted at $2.3 \mathrm{~J}$ (a) and $2.8 \mathrm{~J}$ (b)

\section{Conclusion}

Infrared thermography was used, in the present work, with the twofold function of non-destructive testing technique and of on line surface temperature recorder. Through the surface temperature mapping it was possible to account for delamination and/or fibre breakage occurred during the impact since any temperature increase is due to the impact energy dissipation. In particular, for impact energy $E_{i} \leq 2 \mathrm{~J}$ a small zone around the impact centre undergoes a small heating up displaying a $\Delta T$ of $0.2 \mathrm{~K}, 0.5 \mathrm{~K}$ at maximum for $E_{i}=2 \mathrm{~J}$; this means that the impact caused only microcracks in the matrix. Conversely, as $E_{i}$ reaches the value of $2.3 \mathrm{~J}$ an abrupt temperature increase up to $20 \mathrm{~K}$ is observed starting in the impact zone and moving along the fibres direction; for $E_{i}=2.8 \mathrm{~J}$ the hot zone strengthens in temperature and assumes the appearance of a cross. Of course, the stronger energy dissipation indicates delamination between layers developing through very thin lines and probable fibre breakage in the central part which was struck by the hammer's nose.

Non-destructive tests with lockin thermography show that only the damage occurred at the higher impact energy values $(2.3$ and $2.8 \mathrm{~J})$ is detectable. To a quantitative analysis and through a comparison between phase and thermal images, it comes out that the detected damage (phase images) corresponds to the hottest zones (thermal images).

From a comparison between data obtained with infrared thermography and the acoustic emission recorded by piezo patches a good agreement is found since to an abrupt surface temperature increase corresponds a significant modification of the acoustic signal. Thus it is possible to establish the impact energy value for onset of material damage.

However, it is worth noting that monitoring online the material thermal behaviour, with an infrared imaging device, helps gaining information also on the initiation phase of the impact damage which is generally assumed to coincide with the formation of micro-cracks.

\section{REFERENCES}

[1] Nasa technical reports. Fracture mechanics for composites state of the art and challenges. Available at http://ntrs.nasa. gov/archive/nasa/casi.ntrs.nasa.gov/20080014103_2008013648.pdf

[2] Meola C, Carlomagno GM, "Impact damage in GFRP: new insights with Infrared Thermography", Composites Part A, vol.41, pp. 1839-1847, 2010.

[3] Meola C, Carlomagno GM, Ricci F, Lopresto V, Caprino G, "Investigation of Impact Damage in Composites with Infrared Thermography", 6th NDT in Progress Proceedings, Prague (Czech Republic) P. Mazal ed., pp 175-182, 2011.

[4] Banerjee S, Ricci F, Shish F, Mal AK, "Health Monitoring of Composite Structures Using Ultrasonic Guided Waves". Advanced Ultrasonic Methods for Material and Structure Inspection - Instrumentation and Measurements Series, Edited by Tribikram Kundu, ISTE Ltd 2007; pp. 43-88 ISBN 978-1-905209-69-X, 2007

[5] Meola C, Carlomagno GM, "Infrared thermography to impact-driven thermal effects", Applied Physics A, vol. 96, pp. 759-762, 2009.

[6] Choi HY, Wu HYT, Chang FK. "A new approach toward understanding damage mechanisms and mechanics of laminated composites due to low-velocity impact: part II—analysis”. J Compos Mater 1991;25:1012-38. 
[7] Choi HY, Chang FK, "A model for predicting damage in graphite/epoxy laminated composites resulting from lowvelocity point impact". J Compos Mater 1992;26:2134-69.

[8] Aslan Z, Karakuzu R, Okutan B, "The response of laminated composite plates under low-velocity impact loading" Composite Structures 2003; 59: 119-127.

[9] Carlomagno GM, Meola C, Ricci F, "Infrared thermography and piezoelectric patches for impact damage detection in composite structures”, IWSHM 2011 - International Workshop on Structural Health Monitoring 2011, Stanford University, Stanford, CA, September 13-15, 2011. 